\title{
Circuit Editing and Failure Analysis Applications using a Three-Ion-Beam (Ga, He and Ne) System and Gas Injection System (GIS)
}

\author{
Deying Xia ${ }^{1}$, Huimeng $\mathrm{Wu}^{1}$, Bernhard Geotze ${ }^{1}$, David Ferranti ${ }^{1}$, Lewis Stern ${ }^{1}$ and John Notte ${ }^{1}$ \\ 1. Carl Zeiss Microscopy, LLC, Ion Microscopy Innovation Center, One Corporation Way, Peabody, \\ MA 01960
}

The focused ion beam (FIB) is widely used in semiconductor industries for circuit editing (CE), failure analysis (FA) and nanofabrication. Gallium FIB is most developed for CE and FA for feature size $>10$ $\mathrm{nm}$. In general, after the FIB processing, the structural characterization is performed using SEM. A gas injection system (GIS) is integrated into a FIB or SEM microscope system to mill or deposit the materials in electron or ion induced processing in CE and FA. Ga FIB induced processing cannot fabricate small enough metal or insulator nanostructures to meet the shrinkage of feature size in current semiconductor processing. In the Zeiss NanoFab system, the Ga ion beam is used to perform the fast and large scale milling, and the helium ion beam is used to obtain the high resolution images. In addition, the neon ion beam is used to mill or assist deposition of smaller functional structures. Appropriate precursors for ion induced etching or deposition are introduced via an integrated GIS. In this paper we evaluate applications in CE and FA using a combination of three different focused ion beams.

The first example is the enhanced etching of silicon ( $\mathrm{Si}$ ) for the backside $\mathrm{CE}$ using a $\mathrm{Ga}$ ion beam in combination with $\mathrm{XeF}_{2}$ gas. A relatively thick layer $(\sim 10 \mu \mathrm{m})$ of Si layer was left in a larger pre-etched pit for backside CE. If the Ga ion beam is used alone, it would take much longer time to etch down the metal layer. We used multiple-step Ga milling to minimize typical sidewall re-deposition. It took a dose of $46 \mathrm{nC} / \mu^{2}{ }^{2}$ to etch down to the metal layer as shown in Figure $1 \mathrm{~A}$. With the assistance of $\mathrm{XeF}_{2} \mathrm{gas}_{\text {, it }}$ only took $0.4 \mathrm{nC} / \mu \mathrm{m}^{2}$ to begin exposure of the metal layer as shown in Figure $1 \mathrm{~B}$. The enhancement factor is as high as 100 and it takes much less time, 10 min with $\mathrm{XeF}_{2}$ comparing to 600 min without $\mathrm{XeF}_{2}$. More importantly, we were able to use end-point detection for $\mathrm{CE}$ for this case. Figure $1 \mathrm{C}$ shows that the dose of $0.4 \mathrm{nC} / \mu^{2}$ initiates exposure of the metal layer. This event is apparent from the signal of the secondary electrons collected on the Everhardt Thornley (ET) detector and represents a reliable method to stop etching at the target layer. Figure 1D gives another example of enhanced etching of $\mathrm{SiO}_{2}$ film on $\mathrm{Si}$ substrate with a combination of the neon ion beam and $\mathrm{XeF}_{2}$ gas. From this curves, we can see the enhancement factor is larger than 3 for both thick and shallow $\mathrm{SiO}_{2}$ layer. Figure 1E shows an additional example of neon ion beam milling with high accuracy for front-side CE for milling a box $150 \mathrm{~nm} \times 150 \mathrm{~nm}$ and $400 \mathrm{~nm}$ deep. It is obvious that endpoint information from curve peak and bottom of Figure $1 \mathrm{~F}$ can be obtained for metal and insulator layers. The neon ion beam can be also used to cut the fine metal line with good control in CE and FA applications.

Metal and insulator nanostructures for CE and FA can be deposited with ion beam induced processing. Helium and neon ion beams used in conjunction with precursor chemistries delivered through a GIS deposit fine metal lines such as $\mathrm{Pt}, \mathrm{W}$ and Co for conductive electrical connections for CE. Figure 2A shows helium ion deposited $10 \mathrm{~nm}$ Co lines with $50 \mathrm{~nm}$ pitch on metal fingers. Insulator materials can be deposited from precursors such as TEOS and PMCPS. Figure 2B shows an array of PMCPS squares with $200 \mathrm{~nm}$ thickness deposited using a helium ion beam. Both metal and insulator structures prepared in helium or neon ion beam induced processing exhibit good corresponding properties: ultralow resistivity as $100 \mu \Omega \cdot \mathrm{cm}$ for metal line and ultrahigh resistivity as $10^{13} \Omega \cdot \mathrm{cm}$ for insulator pad. 

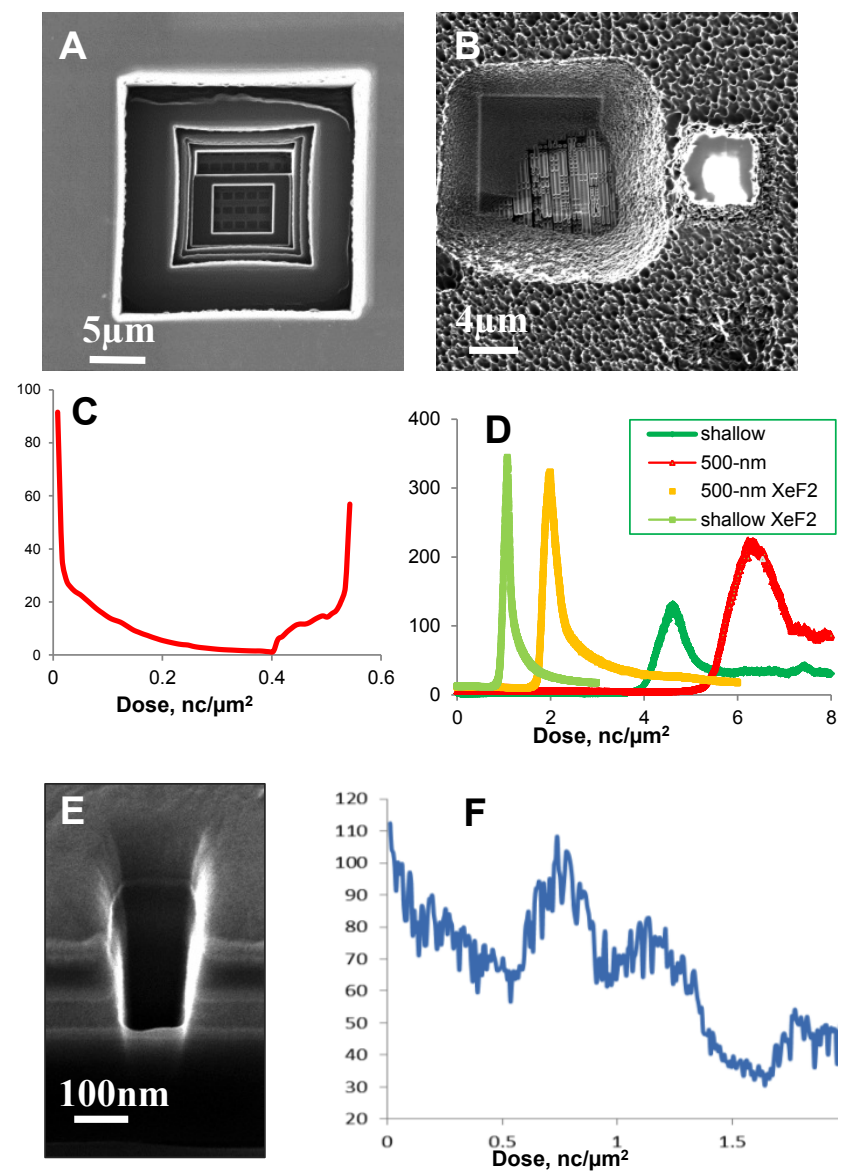

Figure 1. (A)-(B) helium ion image of $\mathrm{Ga}$ ion beam etching Si from backside of circuit (A) multiplestep milling with $\mathrm{Ga}$ ion beam only with total dose $46 \mathrm{nC} / \mu \mathrm{m}^{2}$; (B) $\mathrm{XeF}_{2}$ assisted etching; (C) end-point detection for $\mathrm{XeF}_{2}$-assisted $\mathrm{Si}$ etching for tested chip as in (B); (D) comparison of endpoint detection for with and without $\mathrm{XeF}_{2}$ to etch $\mathrm{SiO}_{2}$ film on $\mathrm{Si}$ substrate using neon ion beam; (E) tilted helium image of etching box on front side of tested chip; (F) endpoint curves for Ne milling processing in (E).
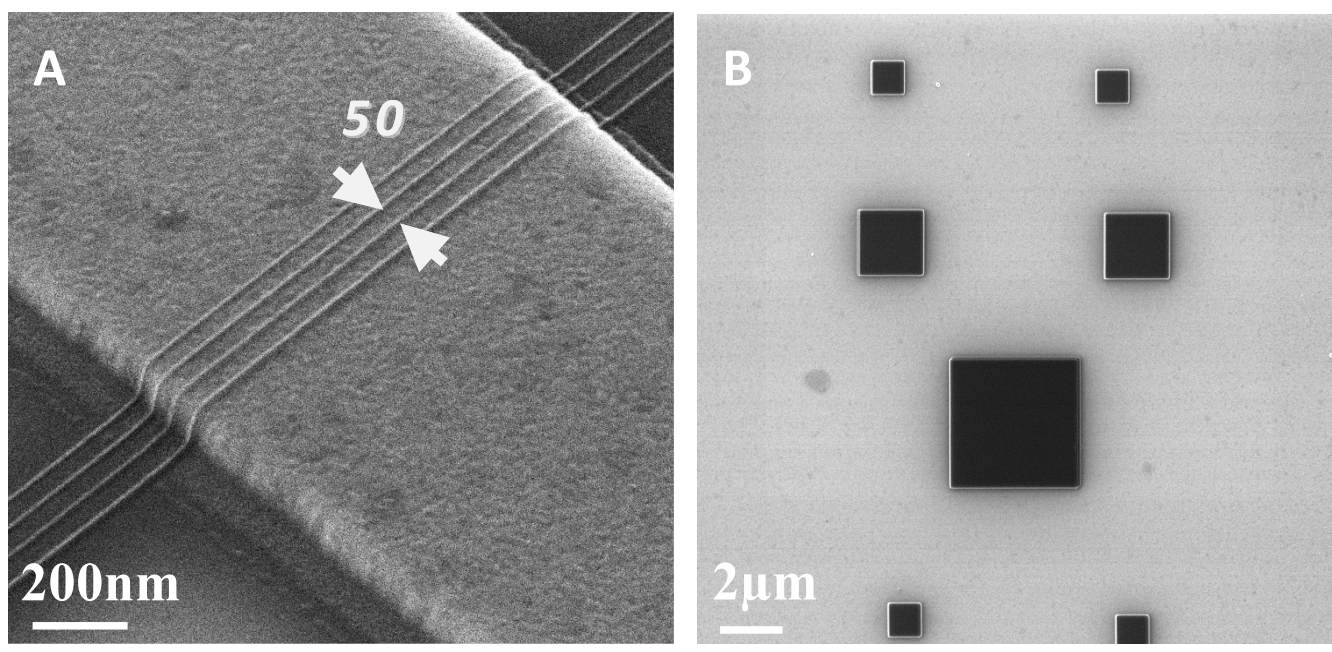

Figure 2. Helium ion image of deposited metal and insulate: (A) tilted view, four Co lines on four finger structures; (B) top view, insulator pad arrays of PMCPS. 